\title{
PAH'S AND SILICATE EMISSION IN NOVA CEN 1986
}

\author{
Craig H. Smith, ${ }^{\dagger}$ David K. Aitken, ${ }^{\dagger} \&$ Patrick F. Roche. ${ }^{\ddagger}$
}

†Dept. of Physics, University College, U.N.S.W, Campbell ACT 2601 Australia. ${ }^{\ddagger}$ Dept. of Astrophysics, Uni. of Oxford, Keeble Rd, Oxford OXQ 3RH England.

We present $8-13 \mu \mathrm{m}$ spectra $(\mathrm{R} \simeq 40$ ) of Nova Cen 1986 (V842 Cen) on 8 occasions between days 162 and 899 after outburst, and all after the onset of dust formation. All observations were made with the UCL cooled grating array spectrometer on the $3.9 \mathrm{~m}$ Anglo-Australian Telescope or the $2.3 \mathrm{~m}$ ANU telescope in Australia. All but the last observation (day 899) show evidence for emission from at least two dust components; a featureless continuum (usually attributed to carbon based dust), a broad emissive component centered on $10 \mu \mathrm{m}$ (probably "silicates"), and the $11.3 \mu \mathrm{m}$ feature from the family of unidentified infra-red dust features (UIR's) seen on days $271-687$.

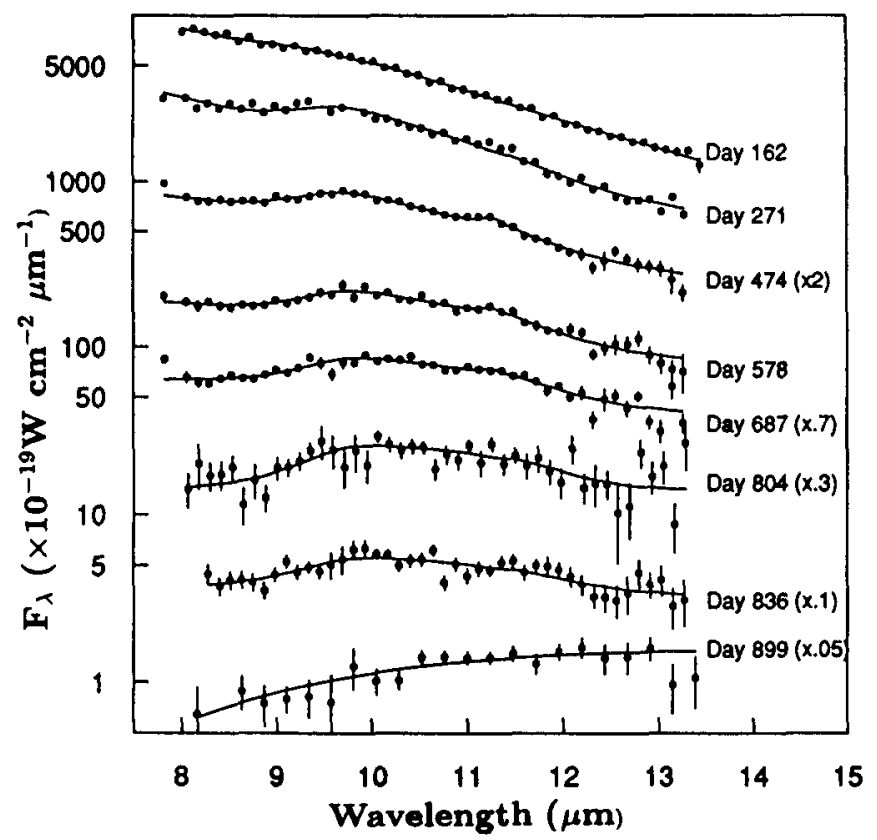

Fig 1: $8-13 \mu \mathrm{m}$ spectra of Nova Cen 1986 taken between day 162 and 899 after outburst. At least two dust components are seen in each spectrum: a featureless continuum (carbon dust) and a broad peak at $10 \mu \mathrm{m}$ (silicate dust); an additional narrow feature at $11.3 \mu \mathrm{m}$ (PAH's or HAC's) is seen between days 271 and 687 . The solid lines represent fits to the spectra. 


\section{Model Fits and Silicate Emission}

We have modelled these spectra with a combination of a featureless (carbon based) continuum, a silicate like emission component, and an $11.3 \mu \mathrm{m}$ UIR feature. Using any $\mathrm{SiC}$ type emissivity curve (i.e.derived from an astronomical source like $\mathrm{Y}$ Tau, or laboratory measurements) gave significantly poorer fits in all cases than did silicates, and so it is unlikely that $\mathrm{SiC}$ grains contribute significantly to the dust emission from this nova. The fits are shown as solid lines in Fig 1, and indicate that during the observed period both dust temperatures fell fairly constantly: from $650 \mathrm{~K}$ to 300 $\mathrm{K}$ for the carbon dust and $450 \mathrm{~K}$ to $200 \mathrm{~K}$ for the "silicate" dust. The emission amplitude of both dust components also fell fairly uniformly, unlike Nova Aql 1982 where the silicate emission fraction grew with time (Roche et al. 1984).

\section{The $11.3 \mu \mathrm{m}$ UIR Feature}

The narrow feature at $11.3 \mu \mathrm{m}$ (detected on days $271-687$ ) is attributed to thermal spiking of tiny carbon grains by UV photons (Sellgren 1983). These tiny grains have been variously identified as polycyclic aromatic hydrocarbon molecules (PAH's) and hydrated amorphous carbon (HAC's). Mitchell \& Evans (1984) predicted that such grains would form in the ejecta of novae, but Nova Cen 1986 is the first nova in which they have actually been detected. Hyland \& McGregor (1988) have also observed the accompanying $3.28 \mu \mathrm{m}$ dust feature in this nova after day 300 . It is not possible to say whether the $11.3 \mu \mathrm{m}$ feature developed after day 162 or was simply masked by the strong continuum emission on this day, although Hyland \& McGregor favour the formation of these tiny grains between day $250-300$. It is also unclear why the $11.3 \mu \mathrm{m}$ emission disappears after day 687: possibly the grains are destroyed, or the UV flux falls to a level too low to stimulate the PAH/HAC emission. The co-existence of both silicate and carbon based dust in the nova ejecta is unusual, and possibly implies separate grain formation periods or $\mathrm{C} / \mathrm{O}$ abundance variations in the ejecta.

\section{References}

Hyland, A.R. \& McGregor, P., 1988, IAU \#135 Interstellar Dust, in press.

Leger, A. \& Puget, J.L., 1983, Astr. Astrophys, 316, L5

Mitchell, R.M. \& Evans, A., 1984, MNRAS, 209, 945

Roche, P.F., Aitken, D.K. \& Whitmore, B., 1984, MNRAS, 211, 535

Sellgren, K., 1983, Astrophys. J., 277, 623 